C. R. Acad. Sci. Paris, t. 332, Série I, p. 1-??, 2001

- PXMA????.TEX -

Rubrique/Heading

(Sous-rubrique/Sub-Heading)

\title{
Equivariant Gerbes over Compact Simple Lie Groups
}

\author{
Kai Behrend ${ }^{a}$, Ping $\mathrm{Xu}{ }^{\mathrm{b}}$, Bin Zhang ${ }^{\mathrm{c}}$ \\ a University of British Columbia \\ E-mail: behrend@math.ubc.ca \\ b Pennsylvania State University \\ E-mail: ping@math.psu.edu \\ c State University of New York at Stony Brook \\ E-mail: bzhang@math.sunysb.edu
}

(Reçu le jour mois année, accepté après révision le jour mois année)

\begin{abstract}
Using groupoid $S^{1}$-central extensions, we present, for a compact simple Lie group $G$, an infinite dimensional model of $S^{1}$-gerbe over the differential stack $G / G$ whose Dixmier-Douady class corresponds to the canonical generator of the equivariant cohomology $H_{G}^{3}(G)$. (C) 2001 Académie des sciences/Éditions scientifiques et médicales Elsevier SAS
\end{abstract}

Gerbes Equivariantes sur les groupes de Lie simples compacts

Résumé. En utilisant des extensions $S^{1}$-centrales de groupoïdes, nous présentons, dans le cas d'un groupe simple compact $G$ un modèle de dimension infinie d'une $S^{1}$-gerbe sur un champ différentiable $G / G$ dont la classe de Dixmier-Douady correspond au générateur canonique de la cohomologie équivariante $H_{G}^{3}(G)$. (C) 2001 Académie des sciences/Éditions scientifiques et médicales Elsevier SAS

\section{Version française abrégée}

Soit $G$ un groupe de Lie compact simple, le groupe de cohomologie équivariante $H_{G}^{3}(G)$ contient un générateur canonique $[\omega+\Omega]$ dont la classe est entière (voir Sec. 3.1. pour la définition de $\omega$ et $\Omega$ ), où $G$ agit sur lui-même par conjugaison. Nous réalisons cette classe en termes d'une extension $S^{1}$-centrale de groupoïdes, ou en tant que la classe de Dixmier-Douady d'une gerbe sur un champ différentiable. Le champ est celui correspondant à la transformation de groupoïde $G \times G \rightrightarrows G$. Cet exemple s'intègre dans la théorie générale développée dans [2, 3]. Notre construction est divisée en deux étapes. La première étape s'inscrit dans le cadre de la géométrie de Poisson: pour une variété de Poisson affine $\mathfrak{g}^{*}$ induite par un 2-cocycle d'algèbre de Lie $\lambda \in \wedge^{2} \mathfrak{g}^{*}$, nous construisons son groupoïde symplectique ainsi qu'une extension $S^{1}$-centrale de groupoïdes. La construction se

Note présentée par First name NAME

S0764-4442(00)0????-?/FLA

(C) 2001 Académie des sciences/Éditions scientifiques et médicales Elsevier SAS. Tous droits réservés. 1 


\section{Running authors}

fait de la manière suivante. Soit $S^{1} \longrightarrow \widetilde{G} \stackrel{\pi}{\longrightarrow} G$ une extension $S^{1}$-centrale au niveau des groupes de Lie. On forme les groupoïdes de transformations $\Gamma\left(=G \times \mathfrak{g}^{*}\right) \rightrightarrows \mathfrak{g}^{*}$ et $R\left(=\widetilde{G} \times \mathfrak{g}^{*}\right) \rightrightarrows \mathfrak{g}^{*}(G$ agit sur $\mathfrak{g}^{*}$ par l'action de jauge (2), tandis que $\widetilde{G}$ agit sur $\mathfrak{g}^{*}$ essentiellement par la même action obtenue par composition avec le morphisme de groupes $\pi: \widetilde{G} \rightarrow G$ ). En considérant $R$ comme sousgroupoïde du groupoïde symplectique $T^{*} \widetilde{G} \rightrightarrows \widetilde{\mathfrak{g}}^{*}$, on peut considérer les images réciproques sur $R$ des formes symplectique et de Liouville sur $T^{*} \widetilde{G}$ et obtenir repectivement une 2-forme fermée $\omega_{R} \in \Omega^{2}(R)$ et une un-forme $\theta_{R} \in \Omega^{1}(R)$. Soit $\widetilde{\pi}: R \rightarrow \Gamma$ la projection naturelle induite par $\pi: \widetilde{G} \rightarrow G$.

THÉORÈME 0.1. -

1. La 2-forme fermée $\omega_{R}$ est basique relativement au $S^{1}$-fibré $R \rightarrow \Gamma$, et donc se projette en une 2-forme fermée $\omega_{\Gamma}$ sur $\Gamma$, c.à.d, $\omega_{R}=\widetilde{\pi}^{*} \omega_{\Gamma}$.

2. $\omega_{\Gamma}$ est symplectique, ce qui est compatible avec la structure de groupoïde de telle sorte que cela définisse une structure de groupoïde symplectique sur $\Gamma$. C'est le groupoïde symplectique de la variété de Poisson affine $\mathfrak{g}^{*}$.

3. $\widetilde{\pi}: R \longrightarrow \Gamma$ est une extension $S^{1}$-centrale de groupoïdes de Lie.

4. $\theta_{R}$ est une forme de connexion de préquantification sur le $S^{1}$-fibré $\tilde{\pi}: R \rightarrow \Gamma$ compatible avec la structure de groupoïde, c.à.d., $\partial \theta_{R}=0$ et $d \theta_{R}=\widetilde{\pi}^{*} \omega_{\Gamma}$.

En d'autres termes, $\theta_{R} \in C_{D R}^{2}\left(R_{\bullet}\right)$ est une pseudo-connexion sur $R$ au sens de [2] dont $\omega_{\Gamma} \in$ $Z_{D R}^{3}\left(\Gamma_{\bullet}\right)$ est la pseudo-courbure.

Pour un groupe de Lie simple compact $G$ d'algèbre de Lie $\mathfrak{g}$, la forme basique sur $\mathfrak{g}$ induit une 2-cocycle naturel d'algèbre de Lie sur l'algèbre de Lie de lacets $L \mathfrak{g}$. En appliquant la construction ci-dessus, on obtient un groupoïde symplectique $\left(L G \times L \mathfrak{g} \rightrightarrows L \mathfrak{g}, \omega_{L G \times L \mathfrak{g}}\right)$ ainsi qu'une extension $S^{1}$-centrale $\widetilde{L G} \times L \mathfrak{g} \rightrightarrows L \mathfrak{g}$ de ce dernier. Ici nous identifions $L \mathfrak{g}$ avec $L \mathfrak{g}^{*}$ par la forme de Killing. L'application d'holonomie Hol $: L \mathfrak{g} \rightarrow G$ induit un morphisme naturel de Morita du groupoïde $L G \times L \mathfrak{g} \rightrightarrows L \mathfrak{g}$ vers $G \times G \rightrightarrows G$, dont le morphisme induit en cohomologie de de Rham envoie $[\omega+\Omega]$ sur la classe $\left[\omega_{L G \times L \mathfrak{g}}\right]$. Nous prouvons donc que :

THÉORÈme 0.2. - Soit $\omega+\Omega$ un 3-cocycle comme ci-dessus, définissant une classe entière dans $H_{G}^{3}(G)$. L'extension $S^{1}$-centrale $\widetilde{L G} \times L \mathfrak{g} \rightrightarrows L \mathfrak{g}$ de $L G \times L \mathfrak{g} \rightrightarrows L \mathfrak{g}$ correspond à une $S^{1}$-gerbe sur le champ $G / G$ de classe de Dixmier-Douady $[\omega+\Omega] \in H_{G}^{3}(G)$.

\section{Introduction}

Let $G$ be a compact simple Lie group, the equivariant cohomology group $H_{G}^{3}(G)$ contains a canonical generator of integer class, where $G$ acts on itself by conjugation. We realize this class in terms of a Lie groupoid $S^{1}$-central extension, or as the Dixmier-Douady class of an $S^{1}$-gerbe over a differential stack. The stack is the one which corresponds to the transformation groupoid $G \times G \rightrightarrows G$. This example fits into the general theory developed in 2, 3. Recall that associated to every Lie groupoid $\Gamma \rightrightarrows M$, there are De Rham cohomology groups defined as follows. Define $\Gamma_{p}=\underbrace{\Gamma \times_{M} \ldots \times_{M} \Gamma}_{p \text { times }}$, i.e., $\Gamma_{p}$ is the manifold of composable sequences of $p$ arrows in the groupoid $\Gamma \rightrightarrows M\left(\Gamma_{1}=\Gamma, \Gamma_{0}=M\right)$. We have $p+1$ canonical maps $\Gamma_{p} \rightarrow \Gamma_{p-1}$ (each leaving out one of the $p+1$ objects involved a sequence of composable arrows), giving rise to a diagram

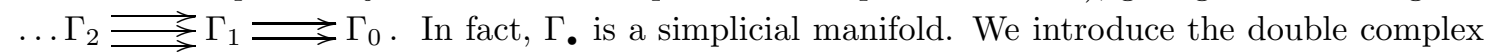

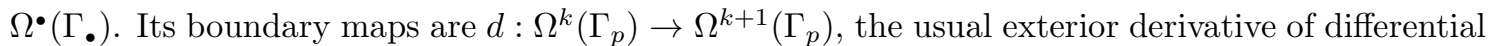
forms and $\partial: \Omega^{k}\left(\Gamma_{p}\right) \rightarrow \Omega^{k}\left(\Gamma_{p+1}\right)$, the alternating sum of the pull back maps of the above diagram. We denote the total complex by $C_{D R}^{*}\left(\Gamma_{\bullet}\right)$ and the total differential by $\delta=(-1)^{p} d+\partial$. The total cohomology groups of $\Omega^{\bullet}\left(\Gamma_{\bullet}\right), H_{D R}^{k}\left(\Gamma_{\bullet}\right)=H^{k}\left(\Omega_{\bullet}\left(\Gamma_{\bullet}\right)\right)$ are called the De Rham cohomology groups 


\section{Running title}

of $\Gamma \rightrightarrows M$. In the case that $\Gamma \rightrightarrows M$ is a transformation groupoid $G \times M \rightrightarrows M$, these are the $G$-equivariant cohomology groups. In [2, we discussed the general question of how to realize a De Rham integer 3-cocycle in terms of an analogue of curvature on a Lie groupoid $S^{1}$-central extension. When this 3-cocycle consists of only one term $\omega \in \Omega^{2}(\Gamma)$, the 3-cocycle condition is equivalent to that $(\Gamma \rightrightarrows M, \omega)$ is a symplectic groupoid if $\omega$ is further assumed to be non-degenerate. $S^{1}$-central extensions of a symplectic groupoid were studied extensively by Weinstein and one of us in [9], to which we refer the readers for details.

When $\Gamma \rightrightarrows M$ is the transformation groupoid $G \times M \rightrightarrows M$, then $\omega+\Omega \in C_{D R}^{3}\left(\Gamma_{\bullet}\right)$, where $\omega \in \Omega^{2}(\Gamma)$ and $\Omega \in \Omega^{3}(M)$, is a 3-cocycle if and only if $d \Omega=0, d \omega=\alpha^{*} \Omega-\beta^{*} \Omega$ and $\left(\partial_{0}^{*}-\partial_{1}^{*}+\right.$ $\left.\partial_{2}^{*}\right) \omega=0$. In this case, the class of $\omega+\Omega$ defines an element in the $G$-equivariant cohomology group $H_{G}^{3}(M)$. When $M=G$ is a compact simple Lie group and $G$ acts on itself by conjugation, an explicit formula for both an $\omega \in \Omega^{2}(\Gamma)$ and an $\Omega \in \Omega^{3}(M)$ appeared in [1] in the study of group valued momentum maps and the moduli spaces of flat connections over two surfaces. See also $[8$ and $[5]$ for the related topics and motivation. However, the fact that $\omega+\Omega$ is a 3 -cocycle in $C_{D R}^{3}\left(\Gamma_{\bullet}\right)$ was overlooked in the literature. In this Note, we reinstate this fact, and show that $[\omega+\Omega]$ is an integer class by constructing an $S^{1}$-gerbe over the stack $G / G$ (also called a $G$-equivariant gerbe over $G$ ) which has $[\omega+\Omega]$ as its Dixmier-Douady class. Our method is to pass from the groupoid $G \times G \rightrightarrows G$ to a Morita equivalent infinite dimensional symplectic groupoid, where an $S^{1}$-central extension can be readily constructed by the methods of Poisson geometry.

Applications of this construction to momentum map theory and twisted K-theory will be discussed elsewhere.

\section{Symplectic groupoids of affine Poisson manifolds}

\subsection{General Construction}

Let $\mathfrak{g}$ be a (finite or infinite dimensional) Lie algebra over $\mathbb{R}$, and $\lambda \in \wedge^{2} \mathfrak{g}^{*}$ a Lie algebra 2cocycle. Let $\tilde{\mathfrak{g}}=\mathfrak{g} \oplus \mathbb{R}$ be the corresponding central extension. Assume that $S^{1} \longrightarrow \widetilde{G} \stackrel{\pi}{\longrightarrow} G$ is a central extension on the level of Lie groups, which exists if $\omega_{G} \in \Omega^{2}(G)$, the left invariant closed two-form corresponding to $\lambda$, is of integer class. It is well-known that the transformation groupoid $\widetilde{\Gamma}\left(=\widetilde{G} \times \widetilde{\mathfrak{g}}^{*}\right) \rightrightarrows \widetilde{\mathfrak{g}}^{*}$, where $\widetilde{G}$ acts on $\widetilde{\mathfrak{g}}^{*}$ by coadjoint action: $\widetilde{g} \cdot \widetilde{\xi}=A d_{\widetilde{g}^{-1}}^{*} \widetilde{\xi}, \forall \widetilde{g} \in \widetilde{G}, \xi \in \widetilde{\mathfrak{g}}^{*}\left(A d_{\widetilde{g}^{-1}}^{*}\right.$ stands for the dual of $\left.A d_{\widetilde{g}^{-1}}\right)$, is a symplectic groupoid. The symplectic structure on $\widetilde{G} \times \widetilde{\mathfrak{g}}^{*}$ is the canonical cotangent symplectic structure when $\widetilde{G} \times \widetilde{\mathfrak{g}}^{*}$ is being identified with $T^{*} \widetilde{G}$ via the right translation.

Denote by $\chi: G \longrightarrow \mathfrak{g}^{*}$ the group 1-cocycle integrating the Lie algebra 1-cocycle $\lambda^{b}: \mathfrak{g} \rightarrow \mathfrak{g}^{*}$, $\left\langle\lambda^{b}(v), u\right\rangle=\lambda(v, u), \forall v, u \in \mathfrak{g}$, where $G$ acts on $\mathfrak{g}^{*}$ by the coadjoint action. We assume that $\chi$ exists, which is true, for instance, when $G$ is simply connected. Since $\widetilde{G}$ is a central extension of $G$, its adjoint action on $\widetilde{\mathfrak{g}}$ descends to an action of $G$ given by $g \cdot(X, t)=\left(A d_{g} X, t+\left\langle\chi\left(g^{-1}\right), X\right\rangle\right), \forall g \in$ $G,(X, t) \in \widetilde{\mathfrak{g}}(\cong \mathfrak{g} \oplus \mathbb{R})$, and therefore the induced coadjoint action is

$$
g \cdot(\xi, t)=\left(A d_{g^{-1}}^{*} \xi+t \chi(g), t\right), \quad \forall g \in G,(\xi, t) \in \widetilde{\mathfrak{g}}^{*}\left(\cong \mathfrak{g}^{*} \oplus \mathbb{R}\right)
$$

Embed $\mathfrak{g}^{*}$ as a hyperplane of $\widetilde{\mathfrak{g}}^{*}$ via the map $\phi: \xi \longrightarrow(\xi, 1), \forall \xi \in \mathfrak{g}^{*}$. Clearly $\mathfrak{g}^{*}$ is a Poisson submanifold of $\widetilde{\mathfrak{g}}^{*}$ with the affine Poisson relation: $\left\{l_{X}, l_{Y}\right\}=l_{[X, Y]}+\lambda(X, Y), \forall X, Y \in \mathfrak{g}$. By Eq. (1), this hyperplane is invariant under the coadjoint action of $G$, on which it takes the form:

$$
g \cdot \xi=A d_{g^{-1}}^{*} \xi+\chi(g), \quad \forall g \in G, \xi \in \mathfrak{g}^{*}
$$

Let $\Gamma$ be the corresponding transformation groupoid $G \times \mathfrak{g}^{*} \rightrightarrows \mathfrak{g}^{*}$. One may also form the transformation groupoid $R: \widetilde{G} \times \mathfrak{g}^{*} \rightrightarrows \mathfrak{g}^{*}$ (here the $\widetilde{G}$-action on $\mathfrak{g}^{*}$ is essentially the same action (2) 


\section{Running authors}

composing with the group morphism $\pi: \widetilde{G} \rightarrow G)$. Then $R \rightrightarrows \mathfrak{g}^{*}$ is a subgroupoid of the symplectic groupoid $\widetilde{\Gamma} \rightrightarrows \widetilde{\mathfrak{g}}^{*}$ under the natural embedding $i:(\widetilde{g}, \xi) \rightarrow(\widetilde{g}, \phi(\xi))$. By $\theta_{\widetilde{\Gamma}}$ and $\omega_{\widetilde{\Gamma}}$, we denote the Liouville one-form and symplectic two-form on $\widetilde{\Gamma}\left(\cong T^{*} \widetilde{G}\right)$ respectively, and set $\omega_{R}=i^{*} \omega_{\widetilde{\Gamma}} \in \Omega^{2}(R)$ and $\theta_{R}=i^{*} \theta_{\widetilde{\Gamma}} \in \Omega^{1}(R)$. By $\widetilde{\pi}: R \rightarrow \Gamma$, we denote the natural projection: $\widetilde{\pi}(\widetilde{g}, \xi)=(\pi(\widetilde{g}), \xi)$, $\forall(\widetilde{g}, \xi) \in \widetilde{G} \times \mathfrak{g}^{*}$.

THEOREM 2.1. -

1. The closed two-form $\omega_{R}$ is basic for the $S^{1}$-bundle $R \rightarrow \Gamma$, and therefore descends to a closed two-form $\omega_{\Gamma}$ on $\Gamma$, i.e., $\omega_{R}=\widetilde{\pi}^{*} \omega_{\Gamma}$.

2. $\omega_{\Gamma}$ is symplectic and compatible with the groupoid structure so that it defines a symplectic groupoid on $\Gamma$. This is the symplectic groupoid of the affine Poisson manifold $\mathfrak{g}^{*}$.

3. $\tilde{\pi}: R \longrightarrow \Gamma$ is a $S^{1}$-central extension of Lie groupoids.

4. $\theta_{R}$ is a prequantization connection form on the $S^{1}$-bundle $\widetilde{\pi}: R \rightarrow \Gamma$ compatible with the groupoid structure, i.e., $\partial \theta_{R}=0$ and $d \theta_{R}=\tilde{\pi}^{*} \omega_{\Gamma}$.

In other words, $\theta_{R} \in C_{D R}^{2}\left(R_{\bullet}\right)$ is a pseudo-connection on $R$ in the sense of [2] with $\omega_{\Gamma} \in Z_{D R}^{3}\left(\Gamma_{\bullet}\right)$ being its pseudo-curvature. Since $\Gamma \rightrightarrows \mathfrak{g}^{*}$ is a transformation groupoid, its De Rham cohomology is the equivariant cohomology $H_{G}^{*}\left(\mathfrak{g}^{*}\right)$, where $G$ acts on $\mathfrak{g}^{*}$ by the gauge action (2). The Lie groupoid $S^{1}$-central extension $R \rightarrow \Gamma$ can be considered as a geometrical model realizing the class $\left[\omega_{\Gamma}\right] \in H_{G}^{3}\left(\mathfrak{g}^{*}\right)$. Indeed, if $\mathfrak{R}$ and $\mathfrak{X}$ are the differential stacks corresponding to the Lie groupoids $R \rightrightarrows \mathfrak{g}^{*}$ and $\Gamma \rightrightarrows \mathfrak{g}^{*}$, respectively, then $\mathfrak{R}$ is an $S^{1}$-gerbe over $\mathfrak{X}$ whose Dixmier-Douady class is equal to $\left[\omega_{\Gamma}\right] \in H_{D R}^{3}(\mathfrak{X})[2,[3]$.

Remark 1. - Note that $R \rightarrow \Gamma$ is indeed the pull back $S^{1}$-central extension of $\widetilde{G} \stackrel{\pi}{\longrightarrow} G$ via the groupoid morphism $\psi$ from $\Gamma\left(=G \times \mathfrak{g}^{*}\right) \rightrightarrows \mathfrak{g}^{*}$ to $G \rightrightarrows$. defined by the natural projection. As a consequence, $\omega_{\Gamma}$ and $\psi^{*} \omega_{G}$ define the same class in $H_{G}^{3}\left(\mathfrak{g}^{*}\right)$ : the Dixmier-Douady class of the $S^{1}$-gerbe $\mathfrak{R} \rightarrow \mathfrak{X}$. It would be interesting to investigate whether this class is non-trivial when $\lambda \in \wedge^{2} \mathfrak{g}^{*}$ is assumed to be a non-trivial 2-cocycle (otherwise it is obvious that $\psi^{*} \omega$ is a trivial class). In the case of loop groups below, one indeed obtains a non-trivial class.

\subsection{Loop Group Case}

We will apply the above construction to the case of loop groups.

Let $(\cdot, \cdot)$ be an ad-invariant non-degenerate symmetric bilinear form on $\mathfrak{g}$. It is well-known that $(\cdot, \cdot)$ induces a Lie algebra 2-cocycle on the loop Lie algebra $\lambda \in \wedge^{2}\left(L \mathfrak{g}^{*}\right)$ defined by [7]:

$$
\lambda(X, Y)=\frac{1}{2 \pi} \int_{0}^{2 \pi}\left(X(s), Y^{\prime}(s)\right) d s, \forall X(s), Y(s) \in L \mathfrak{g} .
$$

By $\widetilde{L g}$ we denote its corresponding Lie algebra central extension. Assume that $\lambda$ satisfies the integrability condition (i.e., the corresponding closed two-form $\omega_{L G} \in \Omega^{2}(L G)^{L G}$ is of integer class). It defines a loop group central extension $S^{1} \longrightarrow \widetilde{L G} \stackrel{\pi}{\longrightarrow} L G$. By identifying $L \mathfrak{g}^{*}$ with $L \mathfrak{g}$ via the bilinear form $(\cdot, \cdot)$, the 1-cocycle $\chi$ admits the form: $\chi(g(s))=g^{\prime}(s) g(s)^{-1}, \forall g(s) \in L G$, and the gauge action (2) becomes

$$
g \cdot \xi=A d_{g^{-1}}^{*} \xi+g^{\prime} g^{-1}, \quad \forall g \in L G, \xi \in L \mathfrak{g} .
$$

This is the standard gauge transformation when $L \mathfrak{g}$ is identified with the space of connections on the trivial bundle over the unit circle $S^{1}$.

As above, we can form the transformation groupoids $\Gamma: L G \times L \mathfrak{g} \rightrightarrows L \mathfrak{g}$ and $R: \widetilde{L G} \times L \mathfrak{g} \rightrightarrows L \mathfrak{g}$, and define $\omega_{R} \in \Omega^{2}(R)$ and $\theta_{R} \in \Omega^{1}(R)$. 


\section{Running title}

According to Theorem 2.1] we see that the closed two-form $\omega_{R}$ is basic and descends to a closed two-form $\omega_{L G \times L \mathfrak{g}}$ on $\Gamma$.

COROLlary 2.2. $\quad-\left(L G \times L \mathfrak{g} \rightrightarrows L \mathfrak{g}, \omega_{L G \times L \mathfrak{g}}\right)$ is a symplectic groupoid integrating the affine Poisson structure on $L \mathfrak{g}$. Moreover, $\widetilde{\pi}: \widetilde{L G} \times L \mathfrak{g} \rightarrow L G \times L \mathfrak{g}$ is a $S^{1}$-central extension of Lie groupoids, on which $\theta_{R} \in C_{D R}^{2}\left(R_{\bullet}\right)$ defines a pseudo-connection with $\omega_{L G \times L \mathfrak{g}}$ being its pseudocurvature.

\section{An $S^{1}$-gerbe over $G / G$}

\subsection{AMM-groupoids}

Let $G$ be a Lie group equipped with an ad-invariant non-degenerate symmetric bilinear form $(\cdot, \cdot)$. Consider the transformation groupoid $G \times G \rightrightarrows G$, where $G$ acts on itself by conjugation. As in [1, we denote by $\theta$ and $\bar{\theta}$ the left and right Maurer-Cartan forms on $G$ respectively, i.e., $\theta=g^{-1} d g$ and $\bar{\theta}=d g g^{-1}$. Let $\Omega \in \Omega^{3}(G)$ denote the bi-invariant 3 -form on $G$ corresponding to the Lie algebra 3 -cocycle $\frac{1}{12}(\cdot,[\cdot, \cdot]) \in \wedge^{3} \mathfrak{g}^{*}$, and $\omega \in \Omega^{2}(G \times G)$ the two-form:

$$
\omega=-\frac{1}{2}\left[\left(A d_{x} g^{*} \theta, g^{*} \theta\right)+\left(g^{*} \theta, x^{*}(\theta+\bar{\theta})\right)\right],
$$

where $(g, x)$ denotes the coordinate in $G \times G$, and $g^{*} \theta$ and $x^{*} \theta$ are, respectively, the $\mathfrak{g}$-valued one-forms on $G \times G$ obtained by pulling back $\theta$ via the first and second projections, and similarly for $x^{*} \bar{\theta}$.

A simple computation leads to

Proposition 3.1. - $\omega+\Omega$ is a 3-cocycle of the De-Rham total complex of the transformation groupoid $G \times G \rightrightarrows G$, and therefore it defines a class in the equivariant cohomology $H_{G}^{3}(G)$.

Remark 2. -

1. When $G$ is a compact simple Lie group with the basic form $(\cdot, \cdot),[\omega+\Omega]$ is a generator of $H_{G}^{3}(G)$. In Cartan model, it corresponds to the class defined by the $d_{G \text {-closed equivariant }}$ 3 -form $\chi_{G}(\xi)=\Omega-\frac{1}{2}\langle\theta+\bar{\theta}, \xi\rangle: \mathfrak{g}^{*} \longrightarrow \Omega^{*}(G), \forall \xi \in \mathfrak{g}^{*}$.

2. In general, given a transformation groupoid $G \times M \rightrightarrows M$ ( $G$ is assumed to be compact), and a $d_{G^{-c l o s e d}}$ equivariant 3-form $\chi_{G}=\Omega+E(\xi)$, where $\Omega \in \Omega^{3}(M)$ is an invariant closed 3-form on $M$ and $E: \mathfrak{g}^{*} \longrightarrow \Omega^{1}(M)$ a $G$-equivariant linear map, an explicit formula for a two-form $\omega \in \Omega^{2}(G \times M)$ can be found [6], using the Bott-Shulman construction, such that $\omega+\Omega \in Z_{D R}^{3}(G \times M \rightrightarrows M)$ defines the same class of $\chi_{G}$.

\section{2. $S^{1}$-central extensions}

Next we want to construct an $S^{1}$-central extension of Lie groupoids which realizes the class of the 3-cocycle $\omega+\Omega$ in Proposition 3.1 as its Dixmier-Douady class. Since $\Omega \in \Omega^{3}(G)$ is not exact, first of all we need to pass to a Morita equivariant groupoid [2]. There are many different choices for such a groupoid. Basically, one needs to choose a surjective submersion $f: M^{\prime} \rightarrow G$ such that the pullback three form $f^{*} \Omega$ is exact on $M^{\prime}$. Then $\Gamma^{\prime}: M^{\prime} \times_{G, \alpha} \Gamma \times_{\beta, G} M^{\prime} \rightrightarrows M^{\prime}$ becomes a groupoid, and the natural projection from $\Gamma^{\prime} \rightrightarrows M^{\prime}$ to $\Gamma \rightrightarrows G$ is a Morita morphism [2]. For instance, one choice is to take a good open cover $\left\{U_{i}\right\}$ of $G$. Another choice, which is the one that we will pursue in this Note, is the infinite dimensional manifold $L \mathfrak{g}$ while $f$ is the holonomy map $\operatorname{Hol}: L \mathfrak{g} \longrightarrow G$, i.e., the time-1 map of the differential equation: $\operatorname{Hol}_{s}(X)^{-1} \frac{\partial}{\partial s} \operatorname{Hol}_{s}(X)=X, \operatorname{Hol}_{0}(X)=e$. Then we have $\mathrm{Hol}^{*} \Omega=d \mu$, where $\mu$ is the two-from on $L \mathfrak{g}: \mu=\frac{1}{2} \int_{0}^{1}\left(\operatorname{Hol}_{s}^{*} \bar{\theta}, \frac{\partial}{\partial s} \operatorname{Hol}_{s}^{*} \bar{\theta}\right) d s[1$.

Proposition 3.2. - 


\section{Running authors}

1. We have a Morita morphism $f$ of Lie groupoids from $L G \times L \mathfrak{g} \rightrightarrows L \mathfrak{g}$ to $G \times G \rightrightarrows G$, which is given by $f(g, r)=(g(0), \operatorname{Hol}(r))$ on the space of morphisms and by $f(r)=\operatorname{Hol}(r)$ on the space of objects, $\forall g \in L G, r \in L \mathfrak{g}$.

2. Under the induced isomorphism $f^{*}: H_{G}^{3}(G) \stackrel{\sim}{\longrightarrow} H_{L G}^{3}(L \mathfrak{g}),[\omega+\Omega]$ goes to $\left[\omega_{L G \times L \mathfrak{g}}\right]$. Indeed we have

$$
\omega_{L G \times L \mathfrak{g}}-f^{*}(\omega+\Omega)=\delta \mu .
$$

As a consequence, we have

THEOREM 3.3. - Let $G$ be a Lie group equipped with an ad-invariant non-degenerate symmetric bilinear form $(\cdot, \cdot)$. Assume that $\lambda \in \wedge^{2}\left(L \mathfrak{g}^{*}\right)$ as in Eq. (3) satisfies the integrability condition. Then the 3-cocycle $\omega+\Omega$ corresponds to an integer class in $H_{G}^{3}(G)$. The $S^{1}$-central extension $\widetilde{L G} \times L \mathfrak{g} \rightrightarrows L \mathfrak{g}$ of $L G \times L \mathfrak{g} \rightrightarrows L \mathfrak{g}$ corresponds to an $S^{1}$-gerbe over the stack $G / G$ with the DixmierDouady class $[\omega+\Omega] \in H_{G}^{3}(G)$.

Remark 3. - If there is a Morita morphism from Lie groupoid $\Gamma^{\prime} \rightrightarrows M^{\prime}$ to $\Gamma \rightrightarrows M$, then these two groupoids are also Morita equivalent in the sense of 10, which means that there is a bimodule. Indeed, these two notions of Morita equivalence are equivalent 3 . Morita equivalence via bimodules is particularly useful in constructing $S^{1}$-central extensions. It allows one to construct the $S^{1}$-central extension of one groupoid in terms of an $S^{1}$-central extension of the other together with a prequantization of the bimodule. See [3] for the details. This, for instance, will lead to a construction of an $S^{1}$-central extension of the Morita equivalent Lie groupoid $\Gamma^{\prime} \rightrightarrows M^{\prime}$ when $M^{\prime}=\cup U_{i}$ is an open covering as in [4, 6] .

We end the paper with the following proposition which explicitly describes the equivalence bimodule between the groupoids $G \times G \rightrightarrows G$ and $L G \times L \mathfrak{g} \rightrightarrows L \mathfrak{g}$.

Proposition 3.4. - The groupoids $G \times G \rightrightarrows G$ and $L G \times L \mathfrak{g} \rightrightarrows L \mathfrak{g}$ are Morita equivalent in the sense of Definition 2.1 in [10], where the bimodule $X$ can be taken as $G \times L \mathfrak{g}$, and $\rho: X \longrightarrow G$ and $\sigma: X \longrightarrow L \mathfrak{g}$ are given, respectively by $\rho(g, r)=g H o l(r) g^{-1}$ and $\sigma(g, r)=r, \forall(g, r) \in G \times L \mathfrak{g}$.

The groupoid $G \times G \rightrightarrows G$ acts on $X$ from the left by: $\left(g_{1}, g_{2}\right) \cdot(g, r)=\left(g_{1} g, r\right), \forall\left(g_{1}, g_{2}\right) \in$ $G \times G, \quad(g, r) \in G \times L \mathfrak{g}$, such that $g_{2}=g \operatorname{Hol}(r) g^{-1} ;$ while $L G \times L \mathfrak{g} \rightrightarrows L \mathfrak{g}$ acts from right: $(g, r) \cdot\left(g(s), r^{\prime}\right)=\left(g g(0), r^{\prime}\right), \forall(g, r) \in G \times L \mathfrak{g},\left(g(s), r^{\prime}\right) \in L G \times L \mathfrak{g}$, such that $r=g(s) \cdot r^{\prime}$.

Acknowledgements. We thank Eckhard Meinrenken and Alan Weinstein for useful discussions, and Pierre Bieliavsky for his help in preparing the French abbreviated version. We also thank the Ecole Polytechnique for hospitality and support of the research summarized in this Note. This research is partially supported by NSF grant DMS00-72171 and NSERC grant 22R81946.

\section{References}

[1] Alekseev, A, Malkin, A, and Meinrenken, E., Lie group valued moment maps, J. Diff. Geom. 48 (1998), 445-495.

[2] Behrend, K. , and Xu, P., $S^{1}$-bundles and gerbes over differential stacks, C.R. Acad. Sci. Paris

[3] Behrend, K. , and Xu, P., Differential stacks and gerbes, in final preparation.

[4] Brylinski, J.-L., Gerbes on complex reductive Lie groups, math.DG/0002158

[5] Huebschmann, J., Guruprasad K., Jeffrey, L., and Weinstein, A., Group systems, groupoids, and moduli spaces of parabolic bundles, Duke Math. J. 89 (1997), 377-412.

[6] Meinrenken, E., The basic gerbe over a compact simple Lie group, math.DG/0209194

[7] Pressley, A., and Segal, G., Loop groups, Oxford University Press, New York, 1986.

[8] Weinstein, A.,, The symplectic structure on moduli space, The Floer memorial volume, Progr. Math., 133 (1995), 627-635.

[9] Weinstein, A., and $\mathrm{Xu}$, P., Extensions of symplectic groupoids and quantization, J. Reine Angew. Math. 417 (1991), 159-189.

[10] Xu, P., Morita equivalent symplectic groupoids, Symplectic geometry, groupoids, and integrable systems, Seminaire sud Rhodanien a Berkeley, 1989 eds by P. Dazord and A. Weinstein, (1991), 291-311. 